\title{
CÓMO CUMPLIR CON LOS ESTÁNDARES DE ACREDITACIÓN: EL PAPEL ESENCIAL DEL DESARROLLO DOCENTE
}

La calidad, o la búsqueda de la misma, es la motivación fundamental de todos los sistemas de acreditación. Si bien son muchos los factores que contribuyen a la calidad de la educación superior -tales como infraestructura física, bibliotecas, tecnología informática y proporción entre catedráticos y estudiantes- el más importante es la calidad del plantel docente. En los últimos cuarenta años, los programas administrados por LASPAU ${ }^{1}$ han contribuido al desarrollo del profesorado en más de 400 universidades de América Latina y el Caribe. A través de esta experiencia, LASPAU ha advertido la existencia de una correlación clara y positiva entre los grandes esfuerzos que realizan las universidades por desarrollar a su profesorado y la calidad general de las instituciones.

La actual economía del conocimiento exige que los graduados universitarios cuenten con un grado de competencia cada vez mayor y que los docentes universitarios dispongan de un alto nivel de destrezas. Este artículo analiza la situación actual en lo que se refiere a la calidad del profesorado universitario en América Latina y el Caribe, examina diversas estrategias para el desarrollo docente y analiza las consecuencias de las mismas en lo que respecta a los esfuerzos de acreditación. Finalmente, pone en tela de juicio algunos de los mitos que se han creado en torno al desarrollo docente.

1 LASPAU (Academic and Professional Programs for the Americas) fue fundado en 1964 en la Universidad de Harvard. Su misión es ofrecer acceso a oportunidades educacionales globales, estimular la creación y transferencia de conocimientos, y promover el entendimiento intercultural en el continente americano. Cumple esta misión por medio de la administración de programas de becas internacionales, incluyendo el Programa de Intercambio Académico Fulbright -dependiente del Departamento de Estado de los Estados Unidos de Norteamérica-, diversas iniciativas de la Organización de Estados Americanos, y una serie de programas auspiciados por gobiernos, fundaciones y corporaciones de América Latina. 


\section{La calidad del profesorado en América Latina y el Caribe}

La calidad de los docentes universitarios en América Latina y el Caribe puede medirse de diversas formas. Este documento examina las dos maneras que más fácilmente se prestan a la cuantificación: el grado de educación alcanzado y la cantidad de investigación producida. En ambas categorías se han logrado grandes avances en los últimos diez años.

\section{Grados de educación}

Los gobiernos de varios países latinoamericanos y caribeños han reconocido la importancia de invertir en el nivel de educación del profesorado que se desempeña en sus universidades nacionales. El Programa para el Mejoramiento del Profesorado (PROMEP) de México, una iniciativa similar implementada a fines de los años noventa por el Ministerio de Educación y Cultura de Argentina, y el Programa de Mejoramiento de la Calidad y Equidad de la Educación Superior (MECESUP) de Chile son tres ejemplos de programas que promueven la educación de posgrado de los docentes universitarios.

En países como Brasil, Colombia y México, los docentes universitarios deben tener estudios de posgrado para lograr la titularidad de una cátedra. Sin embargo, en América Latina y el Caribe el porcentaje total de docentes que cuentan con grado de doctor sigue siendo bajo. Según un artículo publicado en abril de 2005 por Lauritz B. Holm-Nielsen y Kristan Thorn, del Banco Mundial, “...en México y Colombia menos del 4\% de los docentes universitarios tiene doctorado, y sólo uno de cada diez líderes regionales ha obtenido dicho grado académico, con la excepción de Brasil. El nivel de carencia de docentes universitarios altamente calificados también se refleja en el hecho de que en América Latina, en general, el porcentaje de profesores con maestrías es inferior al 26\%". Según Holm-Nielsen y Thorn, el promedio regional de catedráticos con doctorado es sólo del 6\% (Holm-Nielsen y Thorn, 2005). 
En los países con mayores ingresos, el hecho de que sólo unos pocos catedráticos tengan grados académicos avanzados se agrava, porque su distribución al interior del sistema se inclina desmedidamente en favor de las ciudades capitales e instituciones de elite. Las universidades de elite en las grandes ciudades tienden a exhibir mayores porcentajes de docentes con maestrías y doctorados, en tanto que sus contrapartes regionales, en ciudades de importancia secundaria, cuentan con pocos docentes de esta categoría.

Los sistemas de acreditación crean incentivos para mejorar la calidad del profesorado universitario, pero deben ir acompañados de políticas que corrijan los problemas de oferta y distribución. El Instituto Colombiano para el Desarrollo de la Ciencia y la Tecnología (COLCIENCIAS) está invirtiendo en el desarrollo de científicos pertenecientes a universidades regionales. El año pasado inició un programa para formar investigadores en la categoría de doctorado, en que el $85 \%$ de los candidatos provenían de universidades e instituciones de investigación ubicadas fuera de Bogotá, Medellín y Cali. En su diseño el programa estaba dirigido desde el principio hacia estas zonas desplazadas.

Se requieren con urgencia más programas que eleven el número de docentes universitarios con posgrados, en particular, que aborden también el problema de la distribución. MECESUP, en Chile, se concentrará en aumentar la oferta de catedráticos altamente calificados al iniciar su segunda etapa en 2006. Este programa está dirigido a todas las universidades del sistema nacional, en lugar de beneficiar exclusivamente a las instituciones más avanzadas de Santiago y Concepción.

\section{Producción en investigación}

Una segunda forma de medir la calidad de un plantel docente universitario es el número de trabajos científicos que publica en revistas consultadas por sus pares. Según American Scientist, una 
publicación de Sigma Xi, The Scientific Research Society, América Latina ha ostentado la más alta tasa de crecimiento de productividad científica del mundo, medida en función del número de trabajos publicados entre 1991 y 1998. A este incremento han contribuido las nuevas inversiones en investigación realizadas por los consejos nacionales de ciencia y tecnología. En América Latina y el Caribe también han aumentado los proyectos colaborativos internacionales de investigación, gracias a la tasa de participación relativamente alta en Internet que exhibe la comunidad científica.

Sin embargo, a pesar del auge de las publicaciones, la producción total es todavía considerablemente inferior a la de otras regiones del mundo. Por ejemplo, la producción de trabajos científicos en Europa Occidental fue, en ese mismo periodo, catorce veces mayor que la de América Latina. El 85\% de los trabajos publicados provenía de Europa Occidental, América del Norte y la región asiática (Gálvez, Mauqeda, Martínez-Bueno y Valdivia, 2000). Todavía existe una enorme brecha que superar.

\section{Estrategias para el mejoramiento del profesorado}

Si bien se han logrado avances notables, los parámetros de calidad académica señalados anteriormente siguen apuntando a una necesidad de inversión masiva para elevar el nivel educacional del profesorado. El número de docentes con grados académicos avanzados puede aumentarse mediante la creación de incentivos para cursar programas de doctorado con acreditación nacional, el envío a otros países para realizar estudios avanzados, la contratación de académicos extranjeros y la inversión en desarrollo profesional a través de programas de capacitación de docentes "en servicio", tales como la educación ejecutiva.

\section{Estudios en universidades nacionales}

La realización de estudios de posgrado en el propio país se está convirtiendo cada vez más en una excelente estrategia para mejorar 
las credenciales del profesorado. En los últimos diez años las oportunidades de educación de posgrado han aumentado considerablemente en la región. Según la Comisión Fulbright de Chile, en el país se ofrecen más de 477 programas de posgrado, distribuidos en 14 universidades (Saint-Jean, 2003). Estos programas -y otros similares que se ofrecen en Brasil, México, Argentina y Colombia- atraen a estudiantes de maestría y doctorado tanto nacionales como extranjeros, muchos de los cuales optarán por hacer carrera en el mundo académico o en el campo de la investigación.

Los gobiernos de la región están generando incentivos para los estudiantes. El gobierno de Colombia ofrece un crédito financiado por el Banco Mundial para quienes deseen cursar programas de maestría o doctorado. Los consejos nacionales de ciencia y tecnología y los ministerios de educación de diversos países ofrecen atractivas becas para seguir estos programas. Con el MECESUP, Chile es uno de los líderes en esta área.

Las nuevas tecnologías de comunicación y las relaciones de colaboración con universidades internacionales permiten a los programas nacionales de doctorado ofrecer un profesorado mejor calificado y más diverso y una oferta curricular más amplia. Las implicaciones para las universidades son enormes, particularmente en cuanto a su competitividad en el mercado local.

En su reciente obra, The World is Flat, Thomas Friedman destaca el nivel sin precedente de acceso a la información que otorgan las tecnologías de la comunicación. Basta con ingresar al sitio web de cursos libres del Massachusetts Institute of Technology para tener acceso a material de estudio de vanguardia en una amplia gama de disciplinas. En la medida en que aumente el número de países abocados a desarrollar estándares de acreditación que exijan a sus docentes poseer credenciales académicas de posgrado, estos programas nacionales seguirán aumentando y, junto con ello, la oferta de este tipo de educación. 


\section{Programas de intercambio internacional}

Formar profesores en los programas de posgrado locales no debe ser la única estrategia. Por la misma falta de profesores con grados avanzados, muchos de estos programas están limitados en el grado de especialización necesario para enfrentar los nuevos desafíos que plantea la economía del conocimiento. Por lo demás, dado el tamaño que tiene gran parte de los mercados internos, la demanda puede ser suficiente solamente para justificar la existencia de sólo un programa de doctorado, en una disciplina determinada y en todo el país. Si la formación doctoral se centra en un único programa, el nivel de autoincubación académica paralizaría la creatividad.

Una estrategia internacional permite avances cuantitativos de calidad y tiene como beneficios adicionales mayores contactos internacionales y promoción de la investigación y la publicación conjuntas. A los miembros destacados del plantel docente se les debe dar la oportunidad de cursar estudios avanzados en las mejores universidades del mundo.

El elevado costo de los estudios y la pérdida de docentes debido a la fuga de cerebros son dos factores que inhiben a muchas universidades cuando se trata de enviar a sus profesores al exterior para seguir estudios de posgrado. La experiencia de LASPAU demuestra que, con un diseño y ejecución adecuados, estos factores de riesgo se pueden eliminar y la inversión realizada en miembros del plantel docente puede generar retornos significativos.

En el año 2004 LASPAU efectuó una encuesta entre 544 ex becarios del Programa Fulbright de Desarrollo Docente que cursaron estudios en Estados Unidos entre 1976 y 2000 (Morse, Keane, et al., 2003).

Puesto que el programa está diseñado para mantener un contacto estrecho entre los docentes y sus instituciones de origen, los porcentajes de retorno son extremadamente altos. Según la encuesta, tras finalizar sus estudios de posgrado, el 90\% de los 
profesores retornó a sus universidades de origen, el $6 \%$ optó por otro trabajo académico en su propio país y el $4 \%$ aceptó un trabajo en otro.

Con el tiempo, estos docentes se inclinan por permanecer en sus instituciones de origen. Al momento de la encuesta, el 77\% de los docentes permanecía en sus universidades de origen, el 16\% había optado por otro trabajo académico en su propio país y el $4 \%$ había aceptado un trabajo en un país extranjero.

A la inversa de las tendencias predominantes en la región, el $86 \%$ de los ex becarios encuestados que habían retornado a sus instituciones de origen se desempeñaba en cargos de tiempo completo. Adicionalmente, muchos ex becarios habían ascendido a posiciones de liderazgo en sus instituciones como jefes de departamento o como decanos.

La producción científica o académica de los ex becarios Fulbright era impresionante, constituyendo un claro aporte al saber regional y global: el $91 \%$ había realizado presentaciones en conferencias académicas y profesionales (un promedio de ocho exposiciones), el 77\% había publicado artículos académicos o profesionales en revistas sometidas a estudio por sus pares (un promedio de cinco artículos) y el 30\% había publicado libros.

El conocimiento global se enriquece con los estrechos lazos que los ex alumnos mantienen con sus contrapartes estadounidenses. Cerca de dos tercios han emprendido proyectos de colaboración (un promedio de cinco proyectos) con catedráticos o antiguos compañeros de las instituciones anfitrionas.

El Programa de Desarrollo Docente fue diseñado también con el fin de atraer financiamiento estudiantil significativo por parte de las universidades anfitrionas estadounidenses, reduciéndose así el costo general de los estudios de posgrado en el extranjero. 


\section{Contratación de profesores extranjeros}

Muchas universidades contratan profesores en el mercado internacional. El Tecnológico de Monterrey ha tenido mucho éxito con esta estrategia, especialmente cuando es una medida transitoria para reemplazar a sus propios docentes, mientras éstos cursan programas de doctorado en el extranjero. El Tecnológico mantiene oficinas en todo el mundo para atraer académicos. Aplicando una iniciativa similar, cuando la economía argentina entró en proceso de desaceleración entre 1999 y 2000, las universidades chilenas tuvieron la posibilidad de ofrecer excelentes cargos a docentes de ese país en una serie de disciplinas. Si bien la contratación de profesores extranjeros beneficia principalmente a las universidades de elite, es una alternativa viable para aumentar la cantidad y mejorar la calidad de los catedráticos.

\section{Capacitación de docentes en servicio}

La cuarta estrategia es capacitar al profesorado dentro de la misma universidad, especialmente en materias tales como metodología de la enseñanza y de la investigación. A menudo es difícil convencer a los docentes de que los métodos que han aplicado durante los últimos diez años necesitan ser perfeccionados. Pero las nuevas demandas que el mercado impone a las universidades exigen una revisión generalizada de la forma en que enseñan los profesores. Por ejemplo, el estudio de casos y el aprendizaje interactivo gozan de una gran demanda por parte de los estudiantes.

Muchas universidades planean establecer centros de metodología de la enseñanza para garantizar que sus profesores puedan mejorar continuamente sus competencias en este ámbito. Como punto de partida, algunas están invitando a expertos internacionales para realizar talleres sobre nuevos métodos de enseñanza interactiva. LASPAU y el Instituto Centroamericano de Administración de Empresas (INCAE) impartieron recientemente un taller sobre la enseñanza de estudios de casos en la Universidad 
San Pablo (USP-CEU) de España. Actualmente ésta implementa un centro de metodología de la enseñanza que puede elevar significativamente la calidad del profesorado en el corto plazo.

\section{Los mitos en tela de juicio}

Las inversiones destinadas a mejorar la calidad del profesorado son fundamentales si se quiere mejorar la calidad de las universidades. Un funcionario universitario, con quien conversé recientemente, señaló que, incluso si a su universidad le costase US\$100.000 la formación de un docente para la obtención del grado de doctor en una universidad extranjera prestigiosa, dicha inversión sería esencial. Su experiencia ha demostrado que cuando el docente regrese, atraerá varias veces ese monto en fondos de investigación para la universidad.

Otros funcionarios universitarios han expresado su preocupación en cuanto a que sus mejores docentes permanezcan en las universidades y países en los que han cursado sus posgrados. La experiencia citada anteriormente indica que si los programas están debidamente diseñados, los docentes cumplirán los compromisos contraídos con sus instituciones de origen, particularmente si se les anima a crear redes de contactos internacionales y a participar en ellas una vez que retornen a sus países de origen.

El trabajo de LASPAU a lo largo de cuatro décadas demuestra que las grandes brechas de cantidad y calidad que exhiben las universidades en toda la región, medidas en función de las credenciales académicas de sus docentes y de la producción científica de éstos, pueden cerrarse realizando mayores inversiones en los propios miembros del plantel docente. Los estándares de acreditación que incluyan medidas de desarrollo profesional ofrecerán los incentivos necesarios para que las universidades concreten estas inversiones fundamentales. 


\section{Referencias bibliográficas}

Holm-Nielsen, Lauritz B. y Thorn, Kristian. Higher Education in Latin America- A Regional Overview, Banco Mundial, Washington DC, 2005, p. 9.

Gálvez, Antonio; Maqueda, Mercedes; Martínez-Bueno; Valdivia, Eva. Scientific Publication Trends and the Developing World en The American Scientist, Nov. Dic., 2002, Sigma Xi, The Scientific Research Society, Research Triangle Park, Carolina del Norte.

Saint-Jean, Denise. Higher Education in Chile, ponencia presentada en la Reunión Fulbright para América Latina, Punta del Este, Uruguay, 2003.

Adler, Barbash, Henderson de Díaz, Ito-Adler, Keane, Langosy, Morse, Pavese. Fulbright Faculty Development, Ecology, and Cost-Share Programs: A Survey of Alumni 1976-2000, LASPAU: Academic and Professional Programs for the Americas, Cambridge, Massachusetts, 2003. 